\title{
Distribuição vertical e flutuação sazonal da macrofauna vágil associada a Sargassum cymosum C. Agardh, na praia do Lázaro, Ubatuba, São Paulo, Brasil
}

\author{
Giuliano Buzá Jacobucci ${ }^{1}$ \\ Fosca Pedini Pereira Leite ${ }^{1}$
}

\begin{abstract}
Depth distribution and seasonal fluctuations of vagile macrofauna associated with Sargassum cymosum C. Agardh at Lázaro beach, Ubatuba, São Paulo, Brazil. Seasonal fluctuations and depth distribution of the vagile macrofauna associated with Sargassum cymosum at Lazaro beach, Ubatuba, São Paulo State, were evaluated through density comparison of higher taxonomic groups among three depth intervals in four periods of the year. Ten groups were identified and among them gammarid and caprellid amphipods were numerically dominant in all sampling periods and did not show any consistent zonation trend. Gastropods, polychaetes and ophiuroids ocurred in lower densities and tended to dominate as depth increased. Wet weight algae variation did not explain the observed spatial and temporal patterns. Biotic interactions, recruitment and environmental parameters are probably involved in seasonal density variation of the faunal groups studied but it was evident that the depth gradient play an important role in vagile macrofauna vertical distribution.

KEY WORDS. Depth distribution, seasonal fluctuations, macrofauna, Sargassum, phytal, Brazil
\end{abstract}

A comunidade fital associada a macrófitas marinhas é altamente diversa no que se refere à composição faunística e à caracterização das espécięs que a constituem. A estruturação dessas comunidades fitais é influenciada por uma série de fatores ambientais, cuja contribuição relativa pode variar espacial e temporalmente, sendo muitas vezes difícil determinar seus efeitos individuais nas macrófitas e na fauna associada, devido à interação existente entre esses fatores (EDGAR 1983a).

O movimento da água tem sido considerado um dos fatores mais importantes na estruturação da fauna associada a macrófitas (EDGAR \& MOORE 1986), embora os resultados obtidos muitas vezes tenham sido conflitantes. DOMMASNES (1968), estudando o efeito da exposição às ondas na comunidade associada a Corallina officinalis Linnaeus, verificou que a abundância da fauna se modificava tanto devido à variação no crescimento da alga quanto ao hidrodinamismo propriamente dito. Por sua vez, MOORE (1973) concluiu que a quantidade de sedimento depositado entre as frondes de Laminaria hyperborea (Gunn). Fosl. constituía o principal fator de variação da fauna, sendo o efeito direto do movimento da água pouco importante. Diferenças na densidade de vários organismos associados a Amphiroa beauvoisii

1) Departamento de Zoologia, Instituto de Biologia, Universidade Estadual de Campinas. Cidade Universitária, Caixa Postal 6109, 13083-970 Campinas, São Paulo, Brasil. E-mail: jacobucci@ hotmail.com ou fosca@unicamp.br 
Lamouroux, em locais com diferentes graus de agitação e retenção de sedimento pelas algas também foram registradas por MASUNARI (1982), embora a composição específica não tenha sido afetada. GIBBONS (1988) observou diferenças quanto à representatividade de grupos faunísticos da macrofauna com predominância de anfípodes nas praias expostas e maior densidade de isópodes flabelíferos nas abrigadas.

Diferenças de hidrodinamismo podem ocorrer não apenas entre áreas com variação quanto à exposição, mas também em um gradiente de profundidade de um mesmo local. De modo geral, há diminuição da turbulência com a profundidade (HAGERMAN 1966; FENWICK 1976; KRAPP-SCHICKEL 1993) e, em função disto, pode ocorrer zonação tanto qualitativa quanto quantitativa da fauna associada a macrófitas (DAHL 1948; WIESER 1952). Em estudo realizado no sudeste da Tasmânia (EDGAR 1983a), os anfípodes foram mais abundantes até cerca de $2 \mathrm{~m}$ de profundidade, enquanto isópodes e gastrópodes se mostraram proporcionalmente mais representativos abaixo de $5 \mathrm{~m}$.

Esses padrões de distribuição vertical podem ou não ser consistentes ao longo do tempo (GAMBI et al. 1992) devido a alterações sazonais na composição e abundância das macrófitas e da fauna associada decorrentes de fatores bióticos, como competição (CAINE 1977), predação (NELSON 1979), migração (KIKUCHI 1962) e recrutamento (EDGAR 1983c) além de abióticos (temperatura e variação da incidência luminosa, por exemplo). Alterações na densidade da fauna associada podem estar associadas a ciclos de crescimento e diminuição de biomassa das macrófitas, principalmente em regiões temperadas (MUKAI 1971).

No presente estudo a variação de densidade da macrofauna vágil associada a Sargassum cymosum foi quantificada sob os aspectos espacial e temporal, de modo a se avaliar a existência de padrões de distribuição com a profundidade, ao longo das estações do ano.

\section{MATERIAL E MÉTODOS}

\section{Área de estudo}

O estudo foi desenvolvido na Praia do Lázaro, localizada na Enseada da Fortaleza ( $\left.23^{\circ} 30^{\prime} \mathrm{S}, 45^{\circ} 08^{\prime} \mathrm{W}\right)$, município de Ubatuba, Estado de São Paulo. Esta praia tem extensão limitada por pontões do embasamento da Serra do Mar, caracterizando uma praia de bolso, sendo o substrato na região adjacente à praia composto de areia grossa e cascalho, fragmentos biodetríticos e alto teor de matéria orgânica (NEGREIROS-FRANSOZO et al. 1991). O costão nordeste, onde foram realizadas as coletas, situado na extremidade adjacente à praia Sununga, pode ser considerado moderadamente exposto à ação de ondas, segundo critério utilizado por SZÉCHY \& PAULA (1997), estendendo-se por cerca de $30 \mathrm{~m}$ na direção norte-sul. Apresenta bancos conspícuos de algas, com dominância de Sargassum cymosum C. Agardh, que se estendem do infralitoral superior até cerca de $4 \mathrm{~m}$ de profundidade. A identificação de Sargassum se baseou nos registros de ocorrência e caracterização morfológica fornecidos por PAULA (1988). Outras espécies de algas como Dictyopteris delicatula Lamouroux, D. plagiograma (Montagne) Vickers, Bryothamnion 
seaforthii (Turner) Kützing e Gracilaria aff. verrucosa (Hudson) Papenfuss, também ocorrem no local, sob a cobertura de Sargassum, embora em menor abundância (PAULA \& ESTON 1989; SZÉCHY \& PAULA 1997).

\section{Procedimento de amostragem}

A amostragem foi realizada em um setor delimitado paralelamente ao costão com 20 m de extensão. Utilizando-se equipamento de mergulho autônomo, foram coletadas aleatoriamente nove frondes de Sargassum cymosum em cada uma das três faixas de profundidade $0,5-1,5 \mathrm{~m}, 1,5-2,5 \mathrm{~m}$ e 2,5-3 m, delimitadas através de profundímetro digital, em intervalos de três meses em marés equivalentes. A cada coleta sazonal (primavera-outubro 1997, verão-janeiro 1998, outono-abril 1998 e inverno-julho 1998) foram obtidas, portanto, 27 frondes de Sargassum.

No momento da coleta, cada fronde foi individualmente coberta com um saco de tecido com malha de $250 \mu \mathrm{m}$, retendo a macrofauna associada, inclusive os indivíduos jovens. Todas as frondes foram raspadas do substrato juntamente com o apressório, com o auxílio de uma espátula.

\section{Tratamento das amostras}

No laboratório, cada fronde coletada foi lavada separadamente em uma bateria de quatro baldes com água do mar e gotas de formol para que os animais se desprendessem da mesma. Este processo é comprovadamente eficiente e remove cerca de $99 \%$ da epifauna (TAYLOR \& COLE 1994). A água de cada balde foi filtrada em rede com malha idêntica àquela utilizada nos sacos de coleta.

Os animais assim separados foram fixados em álcool a 70\%, sendo posteriormente identificados (filo, classe ou ordem) e contados sob microscópio estereoscópico.

As frondes de Sargassum tiveram seu peso úmido determinado após eliminação do excesso de água com auxílio de uma centrífuga manual, padronizando-se o tempo de secagem (dois minutos sob rotação constante).

\section{Análise dos dados}

A densidade dos grupos taxonômicos da macrofauna foi expressa em número de indivíduos por grama de peso úmido de Sargassum. Com o objetivo de verificar a existência de diferenças na densidade dos taxa e na biomassa das frondes de Sargassum em função da profundidade, foi utilizada análise de variância unifatorial, para cada período de amostragem. As flutuações sazonais da fauna e de Sargassum foram avaliadas obtendo-se a média aritmética das densidades dos taxa e da biomassa das frondes em cada intervalo de profundidade dos quatro períodos e realizando-se, posteriormente, ANOVA unifatorial. As análises de variância foram seguidas do teste de Tukey para comparações múltiplas. Os dados foram avaliados quanto à normalidade e homogeneidade de variâncias e quando necessário, transformações adequadas (ZAR 1996) foram realizadas para satisfazer os requisitos de normalidade, homoscedasticidade e aditividade das análises de variância.

As densidades médias dos taxa em cada intervalo de profundidade e período de amostragem foram avaliadas quanto à dissimilaridade utilizando-se o índice de Sorensen. Esse coeficiente é mais indicado que a distância Euclidiana para a análise 
de dados de comunidades, pois é mais sensível a conjuntos de dados heterogêneos e fornece menos peso a valores extremos das variáveis (PC-ORD 3.20). Foram então construídos dendrogramas modo Q (matriz normal) que determinam a relação entre amostras e modo R (matriz inversa) que definem a relação entre grupos taxonômicos, utilizando-se o método de agrupamento da média aritmética não ponderada (UPGMA) (SNEATH \& SOKAL 1973).

\section{RESULTADOS}

\section{Algas}

Embora o peso úmido de Sargassum cymosum não tenha apresentado variações sazonais significativas (ANOVA log peso: $F_{3,8}=1,178, p>0,05$ ), as frondes coletadas na primavera foram relativamente menores quando comparadas aos demais períodos. Considerando-se cada período separadamente foi observada uma tendência de redução no valor desse parâmetro com o aumento da profundidade (Fig. 1), excetuando-se o mês de julho, no qual não foram observadas diferenças significativas (Tab. I).

\section{Composição}

Foram coletados 126.653 organismos da macrofauna vágil. Dez taxa estiveram representados nas amostras: anfípodes gamarídeos e caprelídeos, isópodes, tanaidáceos, decápodes, gastrópodes, poliquetas, ofiuróides, picnogônidos e sipunculídeos.

\section{Distribuição vertical}

Todos os taxa, exceto os sipunculídeos, estiveram representados nas três faixas de profundidade nas quatro estações do ano, embora com diferenças importantes em sua contribuição relativa.

Anfípodes gamarídeos e caprelídeos foram os organismos mais representativos numericamente. As densidades mínimas dos gamarídeos $(11,46 \pm 6,54$ ind/g) e caprelídeos $(7,04 \pm 4,39 \mathrm{ind} / \mathrm{g}$ ) foram observadas na faixa de $0,5-1,5 \mathrm{~m}$ no mês de janeiro de 1998 , enquanto a densidade máxima de gamarídeos $(106,23 \pm$ $63,25 \mathrm{ind} / \mathrm{g}$ ) ocorreu na faixa de 1,5-2,5 m, em julho de 1998 e a de caprelídeos em outubro $(41,58 \pm 33,87 \mathrm{ind} / \mathrm{g})$, entre 2,5 e $3,5 \mathrm{~m}$. Diferenças significativas entre faixas de profundidade foram observadas em janeiro e julho, mas neste último período os valores de $\mathbf{p}$ foram marginais (Tab. II). Em janeiro a faixa superior apresentou densidades menores que as demais (Fig. 2).

Gastrópodes, poliquetas e ofiuróides ocorreram em densidades menores que os anfípodes e apresentaram uma tendência de aumento de representatividade com o aumento da profundidade.

Os poliquetas tiveram sua densidade mínima $(0,79 \pm 0,65 \mathrm{ind} / \mathrm{g}) \mathrm{em}$ janeiro, na faixa superior de profundidade e a máxima $(6,03 \pm 1,5 \mathrm{ind} / \mathrm{g}) \mathrm{em}$ abril entre $2,5 \mathrm{e}$ $3,5 \mathrm{~m}$. Em janeiro e abril ocorreu aumento nas densidades com a profundidade. Os gastrópodes apresentaram menor densidade $(0,55 \pm 0,34 \mathrm{ind} / \mathrm{g})$ no inverno de 1998 , entre 0,5 e $1,5 \mathrm{~m}$, e maior densidade $(5,4 \pm 4,18 \mathrm{ind} / \mathrm{g})$ no verão, na faixa inferior de profundidade. Densidades distintas entre faixas de profundidade foram observadas em 


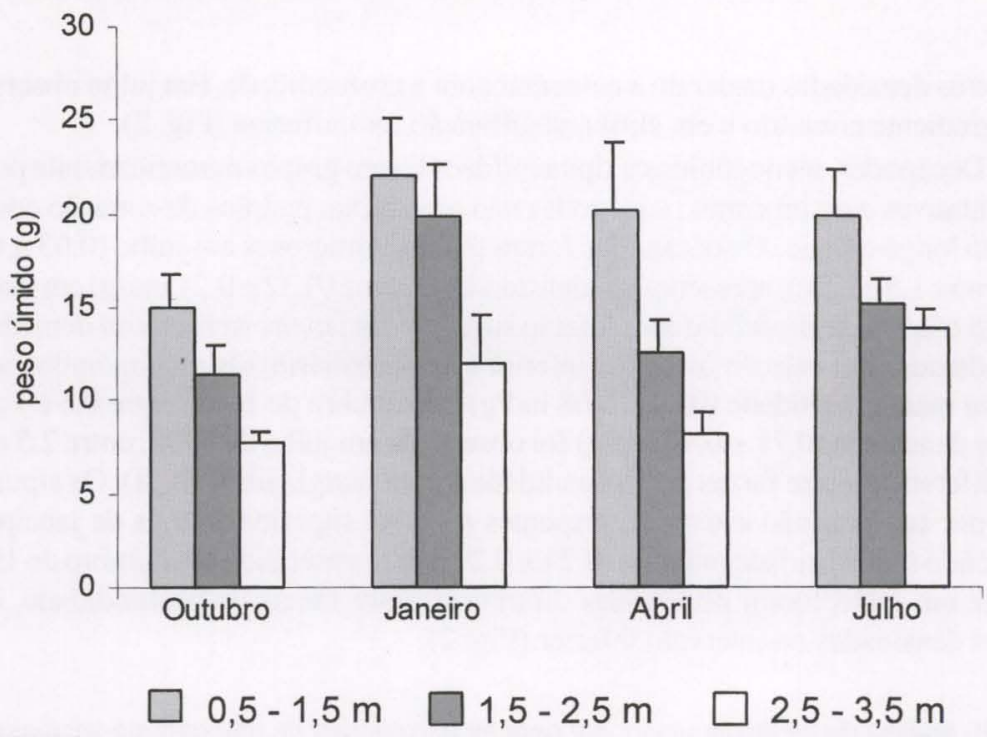

Fig. 1. Peso úmido médio ( \pm erro padrão) das frondes de Sargassum cymosum nos intervalos de 0,5 a $1,5 \mathrm{~m}, 1,5$ a 2,5 $\mathrm{m}$ e 2,5 a 3,5 $\mathrm{m}$ de profundidade nos períodos de outubro de 1997, janeiro, abril e julho de 1998.

Tabela I. Análise de variância unifatorial para o peso úmido de Sargassum cymosum nos intervalos de 0,5 a $1,5 \mathrm{~m}$ (1), 1,5 a $2,5 \mathrm{~m}$ (2) e 2,5 a $3,5 \mathrm{~m}$ (3) de profundidade, para cada período amostrado (intervalos de profundidade unidos por barra horizontal indicam ausência de diferença significativa).

\begin{tabular}{|c|c|c|c|c|c|c|c|c|c|c|c|c|}
\hline & \multicolumn{3}{|c|}{ Outubro $(n=27)$} & \multicolumn{3}{|c|}{ Janeiro $(n=27)$} & \multicolumn{3}{|c|}{ Abril $(n=27)$} & \multicolumn{3}{|c|}{ Julho $(n=27)$} \\
\hline & gl & $\mathrm{F}$ & Tukey & gl & $\mathrm{F}$ & Tukey & gl & $\mathrm{F}$ & Tukey & $g l$ & $\mathrm{~F}$ & Tukey \\
\hline Peso úmido & & \multirow{3}{*}{\multicolumn{2}{|c|}{$5,309^{*} 1$}} & & \multirow{3}{*}{\multicolumn{2}{|c|}{$4,932 * 1$}} & & & & & & \\
\hline Profundidade & 2 & & & 2 & & & 2 & \multicolumn{2}{|c|}{$6,442 * * 1 \overline{23}$} & 2 & \multirow[t]{2}{*}{$2,967^{\text {ns }}$} & 123 \\
\hline Resíduo & 24 & & & 24 & & & 24 & & & 24 & & \\
\hline
\end{tabular}

(ns) Não significativo, $\left({ }^{*}\right) p<0,05,\left({ }^{* *}\right) p<0,01$.

outubro, abril e julho (Tab. II), havendo, a exemplo dos poliquetas, um aumento nas densidades com a profundidade (Fig. 2). A menor densidade $(0,37 \pm 0,66 \mathrm{ind} / \mathrm{g}) \mathrm{de}$ ofiuróides foi registrada em abril na faixa de $0,5-1,5 \mathrm{~m}$ e a maior $(3,10 \pm 3,73 \mathrm{ind} / \mathrm{g})$ em julho na faixa inferior de profundidade. Houve um gradiente de aumento das densidades com a profundidade somente nos meses de outubro e abril.

Os tanaidáceos ocorreram em densidades baixas, mas também apresentaram uma tendência de aumento em suas densidades com a profundidade. A densidade mínima desse grupo $(0,04 \pm 0,05 \mathrm{ind} / \mathrm{g})$ foi observada no mês de abril entre $0,5 \mathrm{e}$ $1,5 \mathrm{~m}$ e a máxima $(1,33 \pm 1,51 \mathrm{ind} / \mathrm{g})$ em janeiro entre 2,5 e $3,5 \mathrm{~m}$.

Os isópodes, presentes em densidades semelhantes aos gastrópodes e poliquetas, apresentaram distribuição vertical diferenciada a cada período de amostragem. A densidade mínima foi registrada em janeiro $(1,34 \pm 1,04 \mathrm{ind} / \mathrm{g})$ na faixa de $0,5-1,5 \mathrm{~m}$ e a mais elevada $(6,93 \pm 3,15 \mathrm{ind} / \mathrm{g})$, no mesmo período entre 2,5 e 3,5 m. Em outubro 
e janeiro as densidades tenderam a aumentar com a profundidade. Em julho observouse um gradiente contrário e em abril a distribuição foi uniforme (Fig. 2).

Decápodes, picnogônidos e sipunculídeos foram grupos numericamente pouco representativos e assim como os isópodes não mostraram padrões de zonação consistentes ao longo do ano. Os decápodes foram menos numerosos em julho $(0,03 \pm 0,05$ $\mathrm{ind} / \mathrm{g})$ entre 1,5 e $2,5 \mathrm{~m}$, apresentando densidade máxima $(0,32 \pm 0,21 \mathrm{ind} / \mathrm{g}) \mathrm{em}$ janeiro entre 1,5 e 2,5 $\mathrm{m}$ de profundidade. A faixa superior em janeiro apresentou densidades mais reduzidas em relação às faixas inferior e intermediária. Os picnogônidos apresentaram menor densidade $(0,04 \pm 0,08 \mathrm{ind} / \mathrm{g})$ em outubro de 1997 , entre 0,5 e $1,5 \mathrm{~m}$. A maior densidade $(0,71 \pm 0,47 \mathrm{ind} / \mathrm{g})$ foi observada em julho de 1998 , entre 2,5 e 3,5 $\mathrm{m}$. As diferenças entre faixas de profundidade foram marginais (Tab. II). Os sipunculídeos, por sua vez, não estiveram presentes na faixa superior do mês de janeiro de

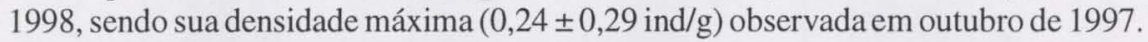
Somente em julho foram observadas diferenças entre faixas de profundidade, com menores densidades no intervalo inferior (Fig. 2).

Tabela II. Análise de variância unifatorial para as densidades da macrofauna vágil associada a Sargassum cymosum nos intervalos de 0,5 a 1,5 m (1), 1,5 a 2,5 m (2) e 2,5 a 3,5 m (3) de profundidade, para cada período amostrado (intervalos de profundidade unidos por barra horizontal indicam ausência de diferença significativa).

\begin{tabular}{|c|c|c|c|c|c|c|c|c|c|c|c|c|}
\hline & \multicolumn{3}{|c|}{ Outubro $(n=27)$} & \multicolumn{3}{|c|}{ Janeiro $(n=27)$} & \multicolumn{3}{|c|}{ Abril $(n=27)$} & \multicolumn{3}{|c|}{ Julho $(n=27)$} \\
\hline & gl & $\mathrm{F}$ & Tukey & gl & $\mathrm{F}$ & Tukey & $\mathrm{gl}$ & $\mathrm{F}$ & Tukey & gl & $\mathrm{F}$ & Tukey \\
\hline \multicolumn{13}{|l|}{ Gammaridea } \\
\hline Profundidade & 2 & \multirow{2}{*}{\multicolumn{2}{|c|}{$0,521^{\mathrm{ns}} 123$}} & 2 & \multirow[t]{2}{*}{8,792 ** } & 123 & 2 & \multicolumn{2}{|c|}{$2,482^{n s} 123$} & 2 & \multirow{2}{*}{\multicolumn{2}{|c|}{$4,516 * 2 \overline{13}$}} \\
\hline Residuo & 24 & & & 24 & & & 24 & & & 24 & & \\
\hline \multicolumn{13}{|l|}{ Caprellidea } \\
\hline Profundidade & 2 & \multicolumn{2}{|c|}{$0,953^{n s} 123$} & 2 & \multirow{2}{*}{\multicolumn{2}{|c|}{$13,671^{* * *} 123$}} & 2 & \multicolumn{2}{|c|}{$2,055^{n s} 123$} & 2 & \multirow{2}{*}{\multicolumn{2}{|c|}{$3,466^{*} 123$}} \\
\hline Resíduo & 24 & & & 24 & & & 24 & & & 24 & & \\
\hline \multicolumn{13}{|l|}{ Isopoda } \\
\hline Profundidade & 2 & \multirow{2}{*}{\multicolumn{2}{|c|}{$12,081^{\text {ns }} 123$}} & 2 & \multirow{2}{*}{\multicolumn{2}{|c|}{$13,716^{\cdots+1}{ }^{2} 3$}} & 2 & \multirow{2}{*}{\multicolumn{2}{|c|}{$0,523^{n s} 123$}} & 2 & \multirow{2}{*}{\multicolumn{2}{|c|}{$4,592 * 1 \overline{23}$}} \\
\hline Residuo & 24 & & & 24 & & & 24 & & & 24 & & \\
\hline \multicolumn{13}{|l|}{ Tanaidacea } \\
\hline Profundidade & 2 & \multirow{2}{*}{\multicolumn{2}{|c|}{$0,382^{n s} 123$}} & 2 & \multirow[t]{2}{*}{$1,434^{\text {ns }}$} & 123 & 2 & \multirow{2}{*}{\multicolumn{2}{|c|}{$11,510^{* * *} 123$}} & 2 & \multirow{2}{*}{\multicolumn{2}{|c|}{$0,283^{n s} 123$}} \\
\hline Residuo & 24 & & & 24 & & & 24 & & & 24 & & \\
\hline \multicolumn{13}{|l|}{ Decapoda } \\
\hline Profundidade & 2 & \multirow{2}{*}{\multicolumn{2}{|c|}{$0,973^{\text {ns }} 123$}} & 2 & \multirow[t]{3}{*}{$5,364^{*}$} & 123 & 2 & $0,155^{*}$ & 123 & 2 & $2,644^{\mathrm{ns}}$ & 123 \\
\hline Residuo & 24 & & & 24 & & & 24 & & & 24 & & \\
\hline Polychaeta & & & & & & & & & & & & \\
\hline Profundidade & 2 & $1,832^{n}$ & 123 & 2 & $3,999^{*}$ & $1 \overline{23}$ & 2 & $17,889 * *$ & *123 & 2 & $0,546^{\mathrm{ns}}$ & 123 \\
\hline Residuo & 24 & & & 24 & & & 24 & & & 24 & & \\
\hline Ophiuroidea & & & & & & & & & & & & \\
\hline Profundidade & 2 & $6,327^{*}$ & 123 & 2 & $1,167^{\text {ns }}$ & 123 & 2 & $5,347^{*}$ & 123 & 2 & $1,843^{\mathrm{ns}}$ & 123 \\
\hline Resíduo & 24 & & & 24 & & & 24 & & & 24 & & \\
\hline Gastropoda & & & & & & & & & & & & \\
\hline Profundidade & 2 & $5,305^{*}$ & 123 & 2 & $0,482^{n s}$ & 123 & 2 & $12,784^{* *}$ & 123 & 2 & $6,829^{*}$ & +123 \\
\hline Resíduo & 24 & & & 24 & & & 24 & & & 24 & & \\
\hline Pycnogonida & & & & & & & & & & & & \\
\hline Profundidade & 2 & $4,226^{*}$ & 123 & 2 & $0,253^{\mathrm{ns}}$ & 123 & 2 & $0,330^{\mathrm{ns}}$ & 123 & 2 & $4,254^{*}$ & $2 \longdiv { 1 3 }$ \\
\hline Resíduo & 24 & & & 24 & & & 24 & & & 24 & & \\
\hline Sipuncula & & & & & & & & & & & & \\
\hline Profundidade & 2 & $0,265^{n}$ & 123 & 2 & $0,937^{\mathrm{ns}}$ & 123 & 2 & $2,145^{\mathrm{ns}}$ & 123 & 2 & $4,764^{*}$ & $1 \overline{23}$ \\
\hline Residuo & 24 & & & 24 & & & 24 & & & 24 & & \\
\hline
\end{tabular}

(ns) Não significativo, $\left({ }^{*}\right) p<0,05,\left(^{* *}\right) p<0,01,\left(^{* * *}\right) p<0,001$. 

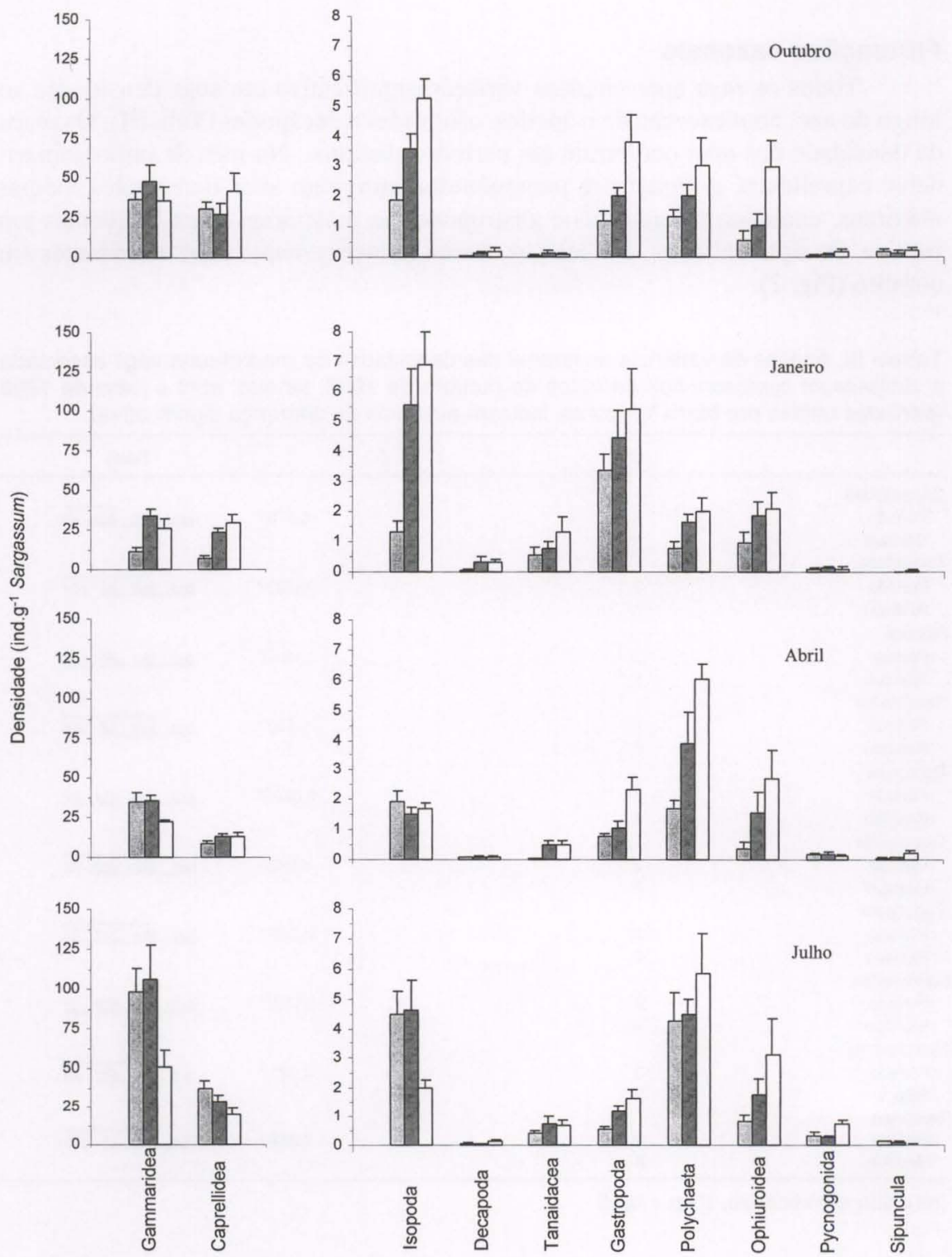

Fig. 2. Densidade média ( \pm erro padrão) de anfípodes gamarídeos e caprelídeos, isópodes, decápodes, tanaidáceos, gastrópodes, poliquetas, ofiuróides, picnogônidos e sipunculídeos associados a Sargassum cymosum nos intervalos de 0,5 a 1,5 m, 1,5 a 2,5 m e 2,5 a $3,5 \mathrm{~m}$ de profundidade nos períodos de outubro de 1997, janeiro, abril e julho de 1998. 


\section{Flutuações sazonais}

Todos os taxa apresentaram variação significativa em suas densidades ao longo do ano, com exceção de isópodes, ofiuróides e decápodes (Tab. III). Os picos de densidade dos taxa ocorreram em períodos distintos. No mês de julho gamarídeos, caprelídeos, poliquetas e picnogônidos atingiram suas densidades médias máximas, enquanto tanaidáceos e gastrópodes se mostraram mais freqüentes em janeiro. Os sipunculídeos, por sua vez, foram numericamente mais abundantes em outubro (Fig. 2).

Tabela III. Análise de variância unifatorial das densidades da macrofauna vágil associada a Sargassum cymosum nos períodos de outubro de 1997, janeiro, abril e julho de 1998 (periodos unidos por barra horizontal indicam ausência de diferença significativa).

\begin{tabular}{|c|c|c|c|}
\hline & gl & $\mathrm{F}$ & Tukey \\
\hline \multicolumn{4}{|c|}{ Gammaridea } \\
\hline Período & 3 & $6,579^{*}$ & jan abr out jul \\
\hline Resíduo & 8 & & \\
\hline \multicolumn{4}{|l|}{ Caprellidea } \\
\hline Período & 3 & $4,102^{*}$ & out $\overline{\text { ian jul } \mathrm{abr}}$ \\
\hline Resíduo & 8 & & \\
\hline \multicolumn{4}{|l|}{ Isopoda } \\
\hline Período & 3 & $1,194^{\mathrm{ns}}$ & out ian abr iul \\
\hline Resíduo & 8 & & \\
\hline \multicolumn{4}{|l|}{ Tanaidacea } \\
\hline Período & 3 & $5,420^{*}$ & out $\overline{a b r \text { iul jan }}$ \\
\hline Resíduo & 8 & & \\
\hline \multicolumn{4}{|l|}{ Decapoda } \\
\hline Período & 3 & $0,889^{\mathrm{ns}}$ & out jan abr jul \\
\hline Resíduo & 8 & & \\
\hline \multicolumn{4}{|l|}{ Gastropoda } \\
\hline Período & 3 & $4,943^{*}$ & jan $\overline{a b r \text { out jul }}$ \\
\hline Resíduo & 8 & & \\
\hline \multicolumn{4}{|l|}{ Polychaeta } \\
\hline Período & 3 & $4,367^{*}$ & jan $\overline{a b r}$ out jul \\
\hline Residuo & 8 & & \\
\hline \multicolumn{4}{|l|}{ Ophiuroidea } \\
\hline Período & 3 & $0,112^{\mathrm{ns}}$ & out jan abr jul \\
\hline Resíduo & 8 & & \\
\hline \multicolumn{4}{|c|}{ Pycnogonida } \\
\hline Período & 3 & $4,781^{*}$ & out jan abr jul \\
\hline Resíduo & 8 & & \\
\hline \multicolumn{4}{|l|}{ Sipuncula } \\
\hline Periodo & 3 & $7,262^{*}$ & out $\overline{a b r \quad j u l ~ j a n}$ \\
\hline Resíduo & 8 & & \\
\hline
\end{tabular}

(ns) Não significativo, $\left({ }^{\star}\right) p<0,05$

\section{Classificação}

A análise de classificação, modo $\mathrm{R}$, dos taxa permitiu distinguir três grupos (Fig. 3A) com índice de dissimilaridade em torno de 0,70. O primeiro grupo constituído pelos gamarídeos e caprelídeos reuniu os taxa mais abundantes em todos os períodos amostrados. O segundo grupo reuniu os taxa com densidades intermediárias, ao longo do ano. Neste grupo, isópodes e gastrópodes foram agrupados, pois ambos apresentaram maiores densidades em janeiro de 1998, estando separados 

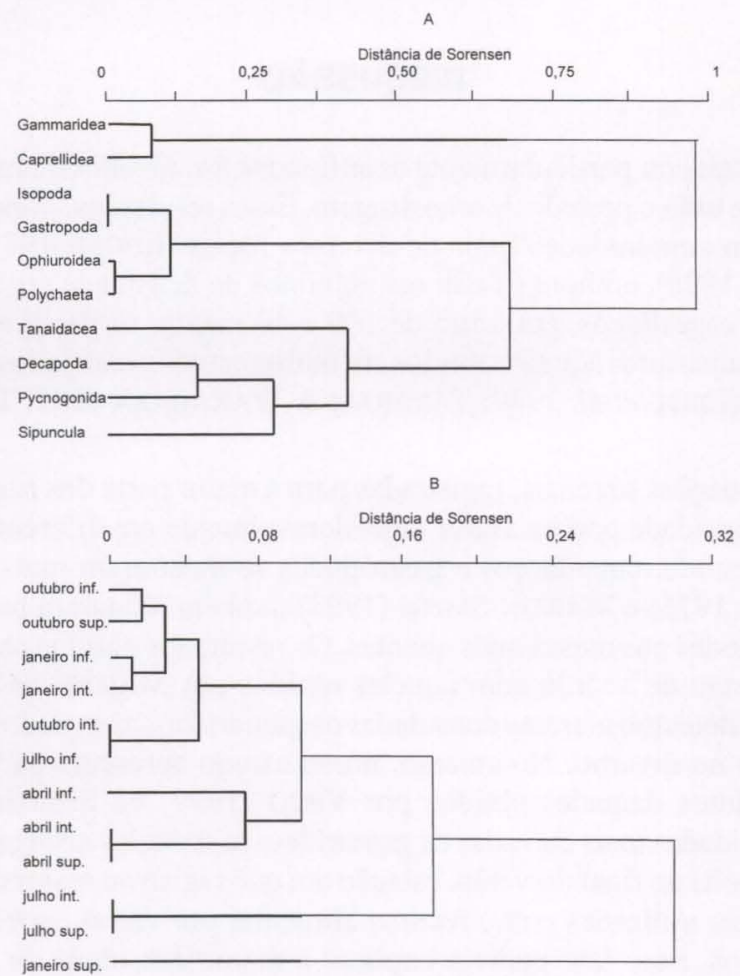

Fig. 3. Dendrogramas da densidade dos taxa (A) e das amostras (B), utilizando-se o índice de Sorensen e o método de agrupamento UPGMA. (sup.: 0,5-1,5 m; int.:1,5-2,5 m; inf.: 2,5-3,5 m).

de ofiuróides e poliquetas que foram mais abundantes em julho (Fig. 3). O terceiro grupo foi constituído pelos taxa com densidades baixas. Dentro deste grupo, os decápodes e picnogônidos foram agrupados, pois apresentaram a mesma distribuição relativa de densidade nos intervalos de profundidade. Em outubro ambos tiveram suas densidades aumentadas com a profundidade, em janeiro e abril foram mais freqüentes entre 1,5 e $2,5 \mathrm{~m}$ e em julho suas densidades foram menores neste mesmo intervalo (Fig. 2).

A análise de classificação, modo Q, das amostras evidenciou quatro grupos com dissimilaridade de 0,10 (Fig. 3B). O intervalo superior de janeiro de 1998 apresentou as menores densidades dos grupos numericamente dominantes, gamarídeos e caprelídeos (Fig. 2). O segundo grupo, constituído pelas amostras superior e intermediária de julho, se separou das demais em decorrência das elevadas densidades de gamarídeos (cerca de 100 indivíduos/g de peso úmido de Sargassum). As amostras de abril constituíram um terceiro grupo, onde os caprelídeos ocorreram em densidades baixas em relação às demais amostras e os poliquetas tiveram representatividade elevada em relação aos anfípodes. Finalmente o quarto grupo foi constituído pelas amostras de outubro, a amostra do intervalo inferior de julho e as amostras de janeiro dos níveis inferior e intermediário. 


\section{DISCUSSÃo}

Os crustáceos, particularmente os anfípodes foram numericamente dominantes ao longo de todo o período de amostragem. Esses resultados, já esperados, foram observados em comunidades fitais de diversos locais (EDGAR 1983b; MASUNARI 1982; DUTRA 1988), embora os valores máximos de densidade registrados para os gamarídeos e caprelídeos, em torno de 100 e 40 inds/g, respectivamente, tenham sido bastante superiores àqueles obtidos em outros estudos realizados no litoral norte de São Paulo (LeITE et al. 1980; TARARAM \& WAKABARA 1981; TARARAM $e t$ al. 1986).

As flutuações sazonais, registradas para a maior parte dos taxa, indicam que os picos de densidade podem variar consideravelmente em diferentes localidades. No presente estudo, tanaidáceos e gastrópodes se mostraram mais freqüentes em janeiro. KITO (1975) e MARTIN-SMITH (1993) também relataram maiores densidades de gastrópodes nos meses mais quentes. Os resultados obtidos para os anfípodes gamarídeos estão de acordo com aqueles obtidos por MARTIN-SMITH (1993), na Austrália, que detectou maiores densidades de gamarídeos associados a três espécies de Sargassum no inverno. No entanto, nosso estudo apresenta dados de variação temporal distintos daqueles obtidos por VIEJO (1999) na Espanha. Essa autora registrou densidades mais elevadas de gamarídeos associados a Sargassum muticum Yendo (Fensholt) no final do verão, estação em que registrou maior disponibilidade de algas epífitas utilizadas como recurso alimentar por várias espécies de gamarídeos herbívoros. Esse fato poderia explicar a maior densidade de gamarídeos na praia do Lázaro em julho, período no qual as frondes de Sargassum apresentaram maior quantidade de algas epífitas (observação pessoal).

Foi possível distinguir dois grupos de organismos, um deles com clara tendência de aumento de densidade com a profundidade, representado por gastrópodes, poliquetas, ofiuróides e tanaidáceos e o outro sem um padrão de zonação batimétrica consistente entre os períodos amostrados, representado pelos demais taxa. O padrão apresentado pelo primeiro grupo poderia ser explicado pelo fato das espécies presentes nestes taxa terem uma resposta semelhante em relação ao gradiente de profundidade ou então serem representados por algumas espécies numericamente dominantes, cuja distribuição vertical caracterizarou o padrão espacial dos grupos taxonômicos como um todo em cada período, não sendo possível evidenciar a distribuição de espécies raras que, por ventura estivessem presentes. A alternância de representatividade numérica entre intervalos de profundidade ao longo do ano do segundo grupo poderia ser atribuída à ocorrência de espécies abundantes com períodos reprodutivos distintos e respostas ambientais características (EDGAR 1983a; SMITH 1996). Isso poderia explicar a grande variação de densidade dos anfípodes nos intervalos de profundidade ao longo do ano já que as espécies de gamarídeos e caprelídeos presentes em áreas adjacentes ao local de estudo apresentam picos reprodutivos e modos de vida bastante variados (LEITE 1996; TARARAM \& WAKABARA 1981). Além disso, tanto as diferenças na densidade dos organismos entre períodos quanto em diferentes escalas espaciais em uma mesma época do ano podem decorrer de características ambientais particulares, 
predação ou interações competitivas (CHAVANICH \& WILSON 2001). Interações bióticas podem restringir a ocorrência das espécies entre praias, entre locais de uma mesma praia e entre períodos do ano (EDGAR 1983a, c). A pressão de predação por peixes e decápodes pode variar espacialmente, na medida em que determinadas áreas podem ser utilizadas para forrageamento com menos freqüência que outras, possibilitando a permanência de algumas espécies (GUNNILL 1982; TAYLOR et al. 1986). Locais com maior complexidade estrutural diminuem a eficiência dos predadores (HOLMLUND et al. 1990; RUSSO 1990), constituindo verdadeiros refúgios para as presas. Além disso, alterações sazonais na abundância de predadores também podem ser responsáveis pelas flutuações das comunidades associadas a macrófitas (EDGAR \& AOKI 1993; SALA 1997).

Aspectos fenológicos, como a biomassa das macrófitas-substrato, também podem ser relevantes para explicar os padrões espaço-temporais da fauna (MUKAI 1971). Embora o peso úmido de Sargassum cymosum não tenha mostrado um padrão evidente de variação sazonal, observou-se que as frondes apresentaram um gradiente de redução de biomassa com a profundidade. Para os grupos que apresentaram aumento de densidade com a profundidade, este é um padrão contrário à relação positiva entre biomassa da alga substrato e abundância da epifauna descrita por alguns autores (STONER \& LEWIS 1985; HECK \& WETSTONE 1977). Isso significa que outros fatores ambientais podem estar envolvidos.

$\mathrm{O}$ aumento de densidade com a profundidade pode estar relacionado a diferenças de hidrodinamismo entre os intervalos de profundidade que, embora não tenham sido quantificadas, podem responder direta ou indiretamente pela distribuição vertical desses taxa. Em profundidades maiores há menor turbulência (FENWICK 1976; KRAPP-SCHICKEL 1993) e, conseqüentemente, maior acúmulo de sedimento nos interstícios das frondes (MOORE 1972). Esse sedimento aumenta a quantidade de substrato disponível para a fauna e microorganismos, elevando, conseqüentemente a disponibilidade de alimento. Além disso, os detritos orgânicos presentes no sedimento também podem constituir importante fonte alimentar para algumas espécies (MASUNARI 1982).

O aumento na dominância de ofiuróides com a profundidade, já foi registrado para a macrofauna associada à alga Ecklonia radiata (C. Ag.) J. Agardh na Austrália (SMITH 1996). Leptochelia savigny (Kroyer, 1842), tanaidáceo tubícola dominante na área de estudo é uma espécie tolerante a distintas condições hidrodinâmicas (PIRES 1980), embora pareça ser favorecida pela presença de sedimento (MASUNARI 1982). Gastropoda foi representado principalmente por Tricolia affinis cruenta Robertson, 1958, um prosobrânquio herbívoro (CRUZ-ABREGO et al. 1994) que reside primariamente entre epífitas e pode ser desalojado sob condições mais intensas de movimento da água, conforme demonstrado experimentalmente por EDGAR \& ROBERTSON (1992).

A distribuição vertical dos poliquetas errantes, representados majoritariamente por espécies de hábitos carnívoros e detritívoros em outras comunidades fitais (MASUNARI 1982; ABBiATI et al. 1987; GAMBI et al. 1992), também sugere a dependência de material depositado sobre as frondes. A confirmação dessa relação, no entanto, só seria possível com a identificação a nível específico dos poliquetas. 
Os resultados obtidos no presente estudo confirmam a importância do fator temporal na estruturação da fauna associada a macrófitas e indicam que a variável profundidade pode desempenhar um papel fundamental na distribuição vertical dos grupos mais representativos da macrofauna vágil associada a Sargassum cymosum.

AGRADECIMENTOS. A A. Güth pelo auxílio nos trabalhos de coleta do material biológico. A A. Turra pelo auxílio nas análises estatísticas. Ao Centro de Biologia Marinha da Universidade de São Paulo (CEBIMar - USP) pelo apoio logístico. À Capes pela concessão da bolsa de mestrado a G.B. Jacobucci durante a execução do projeto e ao CNPq a F.P.P. Leite (Proc. No $300337 / 82-5)$.

\section{REFERÊNCIAS BIBLIOGRÁFICAS}

Abbiati, M.; Bianchi, C.N. \& A. CAStelli. 1987. Polychaete vertical zonation along a littoral cliff in the western Mediterranean. P.S.Z.N.I. Mar. Ecol. 8 (1): 33-48.

CAINE, E.A. 1977. Feeding mechanisms and possible resource partitioning of the Caprellidae (Crustacea: Amphipoda) from Puget Sound, USA. Mar. Biol. 42: 331-33.

ChAvANich, S. \& K.A. WILSON. 2001. Rocky intertidal zonation of gammaridean amphipods in Long Island Sound, Connecticut. Crustaceana 73 (7): 835-846.

Cruz-Abrego, F.M.; Toledano-Granados, A. \& F. Flores-Andolais. 1994. Ecología comunitaria de los gasterópodos marinos (Mollusca: Gastropoda) en Isla Contoy, México. Rev. Biol. Trop. 42 (3): 547-554.

DAHL, E. 1948. On the smaller Arthropoda of marine algae, specially on polyhline waters off the Swedish west coast. Dissertation, Lund. Unders. Over, Øresund 35: 1-193.

DOMMASNES, A. 1968. Variation on the meiofauna of Corallina officianalis L. with wave exposure. Sarsia 34: $117-124$.

Dutra, R.R.C. 1988. A fauna vágil do fital de Pterocladia capillacea (Rodophyta, Gelidiaceae) da Ilha do Mel, Paraná, Brasil. Rev. Brasil. Biol. 48: 589-605.

EDGAR, G.J. 1983a. The ecology of south-east Tasmanian phytal animal communities. I. Spatial organization on a local scale. Jour. Exp. Mar. Biol. Ecol. 70: 129-157.

. 1983b. The ecology of south-east Tasmanian phytal animal communities. II. Seasonal change in plant and animal populations. Jour. Exp. Mar. Biol. Ecol. 70: 159-179.

- 1983c. The ecology of south-east Tasmanian phytal animal communities. IV. Factors afecting the distribution amphitoid amphipods among algae. Jour. Exp. Mar. Biol. Ecol. 70: 205-225.

EDGAR, G.J. \& M. AoKI. 1993. Resource limitation and fish predation: their importance to mobile epifauna associated with japanese Sargassum. Oecologia 95: 122-133.

EdgaR, G.J. \& P.G. Moore. 1986. Macro-algae as habitats for motile macrofauna. Monografias Biológicas 4: 255-277.

EDGaR, G.J. \& A.I. RoberTson. 1992. The influence of seagrass structure on the distribution and abundance of motile epifauna: pattern and process in a western Australian Amphibolis bed. Jour. Exp. Mar. Biol. Ecol. 160: 13-31.

FENwICK, G.D. 1976. The effect of wave exposure on the amphipod fauna of the alga Caulerpa brownii. Jour. Exp. Mar. Biol. Ecol. 25: 1-18.

GAMBI, M.C.; M. LoRENTI; Russo, G.F.; M.B. SCIPIONE \& V.ZUPO. 1992. Depth and seasonal distribution of some groups of the vagile fauna of the Posidonia oceanica leaf stratum: structural and trophic analyses. P.S.Z.N.I. Mar. Ecol. 13 (1): 17-39.

GibBons, M. 1988. The impact of wave exposure on the meiofauna of Gelidium pristoides (Turner) Kuetzing (Gelidiales:Rodophyta). Est. Coast. Shelf Sci. 27: 581-593.

GuNNILL, F.C. 1982. Effects of plant size and distribution on the numbers of invertebrates species and

Revta bras. Zool. 19 (Supl. 1): 87 - 100, 2002 
individuals inhabiting the brown alga Pelvetia fastigiata. Mar. Biol. 69: 263-280.

HAGERMAN, L. 1966. The macro and microfauna associated with Fucus serratus L., with some ecological remarks. Ophelia 3: 1-43.

HECK JR., K.L. \& G. S. WeTSTONE. 1977. Habitat complexity and invertebrate species richness and abundance in tropical seagrass meadows. Jour. Biogeogr. 4: 135-142.

Holmlund, M.; C. H. Peterson \& M.E. Hay. 1990. Does algal morphology affect amphipod susceptibility to fish predation? Jour. Exp. Mar. Biol. Ecol. 139: 65-83.

KIKUCHI, T. 1962. An ecological study on animal community of Zostera belt in Tomioka Bay, Amakusa, Kyushu. II. Community composition (2) Decapod crustaceans. Rec. Oceanogr. Works Japan Spec. 6: 135-146.

Krro, K. 1975. Preliminary report on the phytal animals in the Sargassum confusum region in Oshoro Bay, Hokkaido. Jour. Fac. Sci. Hokkaido Univ., Ser. VI Zool., 20 (1): 141-158.

KRAPP-SCHICKEL, G. 1993. Do algal dwelling amphipods react to the "critical zones" of a coastal slope. Jour. Nat. Hist. 27: 883-900.

Leite, F.P.P.; A.S. Tararam \& Y. Wakabara. 1980. Composição e distribuição da fauna de Gammaridea na região da enseada da Fortaleza - Ubatuba, Estado de São Paulo. Bol. Inst. Oceanogr., São Paulo, 29 (2): 297-299.

LEITE, F.P.P. 1996. Fecundidade de sete espécies de gamarídeos (Crustacea, Amphipoda, Gammaridea) associados à alga Sargassum cymosum. Iheringia, Sér. Zool., 80: 39-45.

MARTIN-SMITH, K.M. 1993. Abundance of mobil epifauna: the role of habitat complexity and predation by fishes. Jour. Exp. Mar. Biol. Ecol. 174: 243-260.

MASUNARI, S. 1982. Organismos do fital de Amphiroa beauvoisii. I. Autoecologia. Bol. Zool., São Paulo, 7: $57-148$.

Moore, P.G. 1972. Particulate matter in the sublittoral zone of na exposed coast and its ecological significance, with special reference to the fauna inhabiting kelp holdfasts. Jour. Exp. Mar. Biol. Ecol. 10: 59-80.

1973. The larger Crustacea associated with holdfasts of kelp (Laminaria hyperborea) in North-East Britain. Cah. Biol. Mar. 14 (4): 493-518.

MUKaI, H. 1971. The phytal animals on the thalli of Sargassum serratifolium in the Sargassum region, with reference to their seasonal fluctuations. Mar. Biol. 8: 170-182.

Negreiros-Fransozo, M; A. Franzoso; M. A. A. Pinheiro; F.L.M. Mantelatto \& S. Santos. 1991. Caracterização física e química da Enseada da Fortaleza, Ubatuba, SP. Rev. Bras. Geoci. 21 (2): 114-120.

NeLSON, W.G. 1979. Experimental study of selective predation on amphipods: consequences for amphipod distribution and abundance. Jour. Exp. Mar. Biol. Ecol. 38: 225-245.

Paula, E.J. 1988. O gênero Sargassum C. AG. (Phaeophyta-Fucales) no litoral do Estado de São Paulo, Brasil. Bol. Bot. Univ. São Paulo 10: 65-118.

PAULA, E.J. \& V.R. EsTON. 1989. Secondary succession on an exposed rocky intertidal algal community of the state of São Paulo, Brazil. Bol. Bot. Univ., São Paulo, 11: 1-9.

PIRES, A.M.S. 1980. Ecological studies on intertidal and infralittoral brazilian Tanaidacea (Crustacea, Peracarida). Stud. Neotr. Faun. Environ. 15: 141-153.

Russo, A.R. 1990. The role of seaweed complexity in structuring Hawaiian epiphytal amphipod communities. Hydrobiologia 194: 1-12.

SALA, E. 1997. The role of fishes in the organization of a Mediterranean sublittoral community. II. Epifaunal communities. Jour. Exp. Mar. Biol. Ecol. 212: 45-60.

SMITH, S.D.A. 1996. The macrofaunal community of Ecklonia radiata holdfasts: variation associated with sediment regime, sponge cover and depth. Austr. Jour. Ecol. 21: 144-153.

SNEATH, P.H.A. \& R.R. Sokal. 1973. Numerical Taxonomy. San Francisco, Freeman and Co., 573p.

STONER, A.W. \& F.G. LEWIS III. 1985. The influence of quantitative aspects of habitat complexity in tropical seagrass meadows. Jour. Exp. Mar. Biol. Ecol. 94: 19-40.

SzÉchy, M.T.M. \& E.J. Paula. 1997. Macroalgas epífitas em Sargassum (Phaeophyta - Fucales) do 
litoral dos estados do Rio de Janeiro e São Paulo, Brasil. Leandra 12: 1-10.

TARARAm, A.S. \& Y. WAKABARA. 1981. The mobile fauna-especially Gammaridea-of Sargassum cymosum. Mar. Ecol. Prog. Ser. 5: 157-163.

TARARAM, A.S.; Y. WAKABARA \& F.P.P. LeITE. 1986. Vertical distribution of amphipods living on algae of Brazilian intertidal rocky shore. Crustaceana 51: 183-187.

TAYLOR, R.B. \& R.G. ColE. 1994. Mobile epifauna on subtidal brown seaweeds in northeastern New Zealand. Mar. Ecol. Prog. Ser. 115: 271-282.

TAYLOR, P.R.; M.M. LITTLER \& D.S. LITTLER. 1986. Escapes from herbivory in relation to the structure of mangrove island macroalgal communities. Oecologia 69: 481-490.

VIEJO, R.M. 1999. Mobile epifauna inhabiting the invasive Sargassum muticum and two local seaweeds in northern Spain. Aquat. Bot. 64: 131-149.

WIESER, W. 1952. Investigations on the microfauna inhabiting seaweeds on rocky coast. IV. Studies on the vertical distribution of the fauna inhabiting seaweeds below the Plymouth Laboratory. Jour. Mar. Biol. Ass. U.K. 31: 145-173.

ZAR, J.H. 1996. Biostatistical Analysis. New Jersey, Prentice-Hall, Inc., $3^{\text {rd }}$ ed., 662p.

Recebido em 29.VI.2001; aceito em 20.V.2002. 